\title{
"Atención a la Diversidad en la Educación Superior: Una propuesta desde el Trabajo Comunal Universitario (TCU)"
}

\author{
Attention to diversity in Higher Education: A proposal from \\ the Community University Work (TCU)
}

MEd. Ligia Quesada Campos*

Resumen: Este artículo se origina en la ponencia presentada en marzo 2017 en el Congreso Internacional sobre Educación Inclusiva en Oviedo, España. Resume los resultados del trabajo realizado en el proyecto TCU-669, Trabajo Comunal Universitario "Atención a la diversidad en el marco de la Educación Inclusiva”. Este proyecto se implementó en el año 2016 como un plan piloto y su objetivo general fue promover acciones que mejoren las condiciones de educación para las personas, en condición de discapacidad y de esta manera coadyuvar en la consecución de metas académicas que mejoren su calidad de vida. Aunque el objetivo del proyecto se planteó en función de los individuos que recibirían el servicio, ha resultado sumamente exitoso porque ha sido una forma de concientizar a los estudiantes universitarios acerca de la diversidad y las diferentes necesidades que pueden tener los seres humanos, además les permite adquirir habilidades deseables para desarrollarse como profesionales en una sociedad diversa. Estudiantes de diferentes carreras se matriculan en este Trabajo Comunal Universitario (TCU), lo que viene a significar para ellos un gran desafío, ya que tienen que desarrollar un sinnúmero de actividades con personas en condición de discapacidad.

Palabras clave: Trabajo comunal universitario(TCU), Atención a la diversidad, Educación Inclusiva, Educación Primaria y Preescolar, Deficiencia.

\begin{abstract}
This article is drawn on a paper presented in March 2017 at the International Congress on Inclusive Education in Oviedo, Spain. It summarizes the results of the work done at TCU-669 (well known as 'TCU' in Spanish), College Community Service, named "Attention to diversity within the framework of Inclusive Education".

This community service was first implemented in 2015 as a pilot plan project, whose objective was to promote actions improving educational conditions for people with disabilities. Consequently, these actions would contribute to the achievement of academic goals to improve people`s quality of life.

Although the aim of the project was based on the individuals who would receive the service, it has been extremely successful in raising awareness and reflection among university students about the diversity and different needs that human beings have. That helps university students acquire desirable skills to develop as future professionals, in a diverse society. Students of different majors enter to this College Community Service meaning a great challenge to work due to the number of different activities in favor of students and people in condition of disability.
\end{abstract}

Key Words: College Community Service (TCU), Attention to diversity, Inclusive Education, Preschool and Elementary Education, person with disability.

* Universidad de Costa Rica. Docente e investigadora, Sede de Occidente. Costa Rica Correo electrónico: ligia.quesada@ucr.ac.cr

Este artículo es la recopilación del trabajo realizado en el Trabajo Comunal Universitario 669: “Atención a la Diversidad en el Marco de la Educación Inclusiva”, desarrollado en la Sede de Occidente a partir del año 2016. 


\section{Introducción}

El proyecto Trabajo Comunal Universitario (TCU) Atención a la diversidad en el marco de la Educación inclusiva ha tenido como propósito abrir espacios para que todas las personas, independientemente de la naturaleza y el grado de discapacidad que presenten, puedan ejercer su derecho de acceso a la educación y desarrollen al máximo su personalidad, sus destrezas, su talento, su creatividad y sus aptitudes o capacidades intelectuales y físicas con el fin de propiciar una mejor calidad de vida, mayores oportunidades educativas y de aprendizaje, a través del apoyo escolar.

Las actividades que se han realizado son: apoyo en el aprendizaje a estudiantes (niños, niñas en edad preescolar y primaria) que presenten algunos problemas de aprendizaje dándoles acompañamiento y reforzamiento en aquellos contenidos que sea necesario. También se han programado actividades artísticas y culturales con esta población para que desarrollen habilidades para la vida que les permita involucrarse en la comunidad y se han realizado talleres con diferentes grupos de primaria y preescolar para promover la inclusión y el respeto al otro. Otro sector de la población que se tomó en cuenta fueron los adultos mayores del Hogar de Ancianos del cantón de San Ramón, a esta población se le ha dado acompañamiento con actividades educativas y culturales para promover un mejoramiento en su calidad de vida. La participación de los estudiantes universitarios a través de la realización de este Trabajo Comunal Universitario (TCU) ha significado una experiencia indispensable y trascendental no solo para las personas beneficiadas, sino para ellos, ya que sus actitudes y valores han sido transformados.

\section{Marco Teórico}

\section{Educación inclusiva}

En nuestro país, aunque se han dado grandes avances por procurar una educación de calidad para todos, donde se visualice la inclusión y se le den apoyos a aquellos estudiantes que presentan condiciones “diferentes” a los demás, es común encontrar en las aulas niños, niñas y jóvenes que son excluidos de la vida escolar y comunitaria por su discapacidad física 
o cognitiva o por presentar problemas de disciplina, superdotación o condiciones socioeconómicas difíciles que no les permite desarrollar un proceso de aprendizaje igual al de sus compañeros.

Comúnmente el concepto, Educación Inclusiva, se asocia con la participación de personas en condición de discapacidad en las instituciones educativas o de otros estudiantes etiquetados como "estudiantes con necesidades educativas", sin embargo esta acepción está más relacionada con el concepto de integración y no el de inclusión. Para mayor claridad será de relevancia hacer la diferenciación entre estos dos vocablos, integración e inclusión.

En la integración, que parte del paradigma médico o rehabilitador, los estudiantes con alguna deficiencia deben adaptarse al sistema, los apoyos que se les brinda parten de sus carencias, solo se insertan personas que se consideran "aptas", no se respeta la individualidad de cada individuo y se trabaja con una metodología que homogeniza y determina que todas las personas aprenden igual y a un mismo ritmo. Desde esta perspectiva, se hacen ajustes y adaptaciones solo para aquellos estudiantes etiquetados "como especiales", dejándose de lado el resto, que también forman parte de la diversidad, que aprenden diferente, porque son diferentes.

Por su parte la inclusión es un concepto mucho más amplio donde toda persona, independientemente de su capacidad, su étnia, su condición socioeconómica o su funcionalidad tiene derecho a ser comprendido en su singularidad, implica que toda persona tiene derecho no solo a estar escolarizada en un aula regular, sino que además deben alcanzar el éxito escolar, gracias a una educación fundamentada en sus habilidades.

El concepto de educación inclusiva surge con la necesidad de romper con prácticas tradicionales que se venían dando en las diferentes instituciones, donde se daba atención únicamente a aquellos estudiantes que, en teoría presentaban alguna necesidad educativa, enfatizándose en la deficiencia, de esta manera se ignoraba las diferentes habilidades que todo individuo tiene.

Al respecto Arnaiz (2003, p. 14) explica que la educación inclusiva:

(...) fue vista en un primer momento como una innovación de la Educación Especial, pero progresivamente se ha ido extendiendo a todo el contexto educativo, como un intento de que la educación, y una educación de calidad, lleguen a todos. (...) no discrimina la discapacidad, la cultura y el género; implica a todos los alumnos de una 
comunidad educativa sin ningún tipo de excepción; todos los estudiantes tengan el mismo derecho a acceder a un currículum culturalmente valioso a tiempo completo como miembros de un aula acorde con a su edad; y enfatiza la diversidad más que la asimilación.

La educación inclusiva se convierte de esta manera en un camino diseñado para facilitar el aprendizaje exitoso y de calidad para todos los niños, jóvenes y adultos que les permita alcanzar sus metas y sueños, disminuir y superar todo tipo de exclusión y hacer valer el derecho de toda persona a una educación accesible, participativa y de excelencia.

\section{Marco legal de la Educación Inclusiva}

La Educación inclusiva ha recorrido un amplio camino, desde la antigüedad, pasando por diferentes paradigmas y modelos. Desde los derechos humanos, la UNESCO, ha procurado normar los aspectos relacionados con la educación para atender y garantizar el disfrute de estos derechos para todas las personas.

La Declaración Universal de los Derechos Humanos escrita por la Organización de la Naciones Unidas establece 30 derechos humanos inalienables para todas las personas, entre ellos el Derecho a la Educación (artículo 26), el cual determina que: Toda persona tiene derecho a la educación, la cual debe ser gratuita y obligatoria, al menos en lo concerniente a la instrucción elemental.

Con base en este derecho, todos los estudiantes, independientemente de su condición, deberán recibir una educación de calidad, donde se atiendan sus necesidades de manera oportuna, para ello todos los miembros de la comunidad educativa deberán prepararse para abrir sus puertas y cumplir con este derecho.

Para tener una mayor claridad con respecto a la legislación que ha coadyuvado en el mejoramiento de la atención a las diferentes necesidades, se hace necesario hacer un poco de historia.

En 1990, se reunieron en Jotiem (Tailandia) representantes de 155 países y 150 organizaciones en un congreso mundial llamado "Educación para todos" (Eduation for All EFA), este congreso fue organizado por la Organización de las Naciones Unidas para la Educación, la Ciencia y la Cultura (UNESCO) y otras organizaciones de gran relevancia 
como el Programa de las Naciones Unidas para el Desarrollo (UNDP), el Fondo de Población de las Naciones Unidas (UNFPA), el fondo de las Naciones Unidas para la Infancia (UNICEF) y el Banco Mundial. Los estados participantes en este congreso se comprometieron a utilizar los recursos internacionales para el mejoramiento de la educación primaria y para reducir el analfabetismo.

Posteriormente, en 1994, se realiza en Salamanca, España el "Congreso Mundial sobre Necesidades Educativas Especiales, Acceso y Calidad; en esta actividad participaron delegados de más de noventa países y veinticinco organizaciones. Este congreso dio como resultado la redacción y publicación del documento: "Declaración de Salamanca y Marco de Acción para las Necesidades Educativas Especiales", entre los aspectos más importantes de este acuerdo, cabe señalar:

- todos los niños tienen derecho fundamental a la educación y debe dárseles la oportunidad de alcanzar y mantener un nivel aceptable de conocimientos,

- cada niño tiene características, intereses, capacidades y necesidades de aprendizaje diferentes, por lo tanto, los sistemas educativos deben ser diseñados y los programas adaptados tomando en cuenta todas las diferencias, características y necesidades,

- las personas con necesidades educativas especiales deben tener acceso a las escuelas ordinarias, que deberán integrarlos con una pedagogía centrada en el niño, capaz de satisfacer esas necesidades,

- las escuelas ordinarias con orientación integradora representan el medio más eficaz para combatir las actitudes discriminatorias, crear comunidades de acogida, construir una sociedad integradora y lograr una educación para todos, además se deberá proporcionar una educación efectiva para la mayoría de los niños y mejorar la eficiencia entre el costo y la eficiencia de todo el sistema educativo.

A raíz de esta convención, en Costa Rica se plantea la Ley 7600 LEY DE IGUALDAD DE OPORTUNIDADES PARA LAS PERSONAS CON DISCAPACIDAD, esta ley fue publicada el 29 de mayo de 1996 y vino a proponer cambios drásticos en estructura en las diferentes instituciones, trato equitativo para todo individuo sin importar su condición y educación de calidad para todos. Desde el artículo 14 hasta el artículo 19 
referidos a la educación se propone un mejoramiento en todo el sector educativo, por ejemplo, mencionan que todas las personas independientemente de su discapacidad tienen derecho al acceso a la educación, a programas educativos que atiendan sus necesidades. También se menciona que todo individuo con alguna necesidad educativa especial puede participar de los programas educativos sin ser excluido de ninguna actividad.

En diciembre de 2006 se firma la "Convención sobre los derechos de las personas con discapacidad y su Protocolo Facultativo" en la Sede de las Naciones Unidas en Nueva York. El texto fue aprobado por el General de Campo y de la ciudad y quedó abierto a la firma el 30 de marzo de 2007. Tras su aprobación, esta Convención fue abierta y ratificada para su aplicación por 192 estados miembros y entró en vigor el 3 de mayo de 2008. Se trató del primer instrumento amplio de derechos humanos del siglo XXI y la primera convención de derechos humanos que se abre a la firma de las organizaciones regionales.

Este documento señala un "cambio paradigmático" de las actitudes y enfoques respecto de las personas con discapacidad y se reafirma que todas las personas, independientemente de su condición, deben poder gozar de todos los derechos humanos y libertades fundamentales. Se aclara y precisa cómo se aplican a las personas con discapacidad todas las categorías de derechos y se indican las esferas en las que es necesario introducir adaptaciones para que puedan ejercer en forma efectiva sus derechos.

El 29 de setiembre de 2008, la Asamblea Legislativa de Costa Rica aprueba y ratifica la convención, a través del decreto ejecutivo 34780, y se establece como la ley 8661. En esta ley se reconocen los derechos que tienen las personas en condición de discapacidad y se determina que, el gobierno de Costa Rica, como estado parte de la Organización de las Naciones Unidas, apoyará y velará porque los acuerdos establecidos en la "Convención sobre los derechos de las personas con discapacidad y su Protocolo Facultativo" se cumplan obligatoriamente, ya que estos acuerdos contribuirán significativamente a paliar la profunda desventaja social de estas personas y promoverá su participación en igualdad de oportunidades, en los ámbitos civil, político, económico, social y cultural. El artículo 24 de esta Convención puntualiza de manera clara los derechos en el campo educativo que tiene toda persona en condición de discapacidad.

Por otra parte, en el Código de la Niñez y la Adolescencia de nuestro país se encuentra un apartado que hace énfasis a la educación, tiene 17 artículos sobre los derechos y deberes 
de los niños, las niñas y adolescentes en la educación. Entre los artículos se resalta el artículo 56 que menciona el derecho a recibir una educación que desarrolle sus potencialidades. En el artículo 58 se encuentran diversos puntos, entre ellos están el de garantizar una educación de calidad y en igualdad de condiciones, el de estimular en todos los niveles el desarrollo del pensamiento autónomo, crítico y creativo, respetando la iniciativa y las características individuales del alumnado, además de propiciar la inclusión.

Aunado a ello, el artículo 62 hace referencia a la educación especial, cita que las personas con un potencial intelectual superior al normal o con algún grado de discapacidad, tendrán el derecho de recibir atención especial en los centros educativos, para adecuar los métodos de enseñanza a sus necesidades particulares. Por último, el artículo 69 menciona que se prohíbe practicar o promover, en los centros educativos, todo tipo de discriminación por género, edad, raza u origen étnico o nacional, condición socioeconómica o cualquier otra que viole la dignidad humana. (Código de la Niñez y Adolescencia).

Cabe señalar también que el Ministerio de Educación Pública, en el documento Plan de Acción de la Educación para Todos 2003-2015”, establece, entre las principales políticas educativas que deben prevalecer en el marco de la educación costarricense especialmente en I, II y III ciclos", la necesidad de que Costa Rica amplíe la cobertura y calidad de la educación, ya que esta se visualiza como la herramienta principal para el mejoramiento de las condiciones de vida de sus habitantes, especialmente aquellos que viven en condiciones de pobreza y exclusión. La educación debe procurar el mejoramiento de las condiciones de equidad y calidad de vida de todos y para ello debe responder adecuadamente, a las nuevas realidades. En el Informe del Estado de la Educación (2013), se señala la necesidad de que Costa Rica supere la distinción entre la cobertura y calidad y exigencias que el país enfrenta en materia social, económica, política y ambiental.

De esta manera la educación inclusiva supone una actitud y un compromiso de contribuir con una educación de calidad, equitativa y justa para todos. Tiene que ver con todos los niños, niñas, jóvenes y adultos con alguna deficiencia, entendida esta como un trastorno físico de cualquier índole o una situación social o económica que le obstaculice su inserción plena en el sistema educativo. Se centra en la presencia, participación real y logro 
en términos de resultados valorados; implica combatir cualquier forma de exclusión; y se considera un proceso que nunca se da por acabado.

El proyecto TCU “Atención a la Diversidad en el marco de la Educación Inclusiva” es totalmente coherente con las políticas inclusivas que promocionan una educación de calidad, sin discriminación de ninguna naturaleza y coincide además con las principales políticas de la Universidad de Costa Rica, en las que se destaca el carácter humanista y transformador de la sociedad para contribuir con el bien común, y de esta manera favorecer el pleno acceso a toda persona en cualquier condición a una educación de calidad.

\section{Desarrollo de la práctica experiencia}

Aunque este proyecto de TCU no constituye propiamente un trabajo de investigación, se podría afirmar que se enmarca dentro de un paradigma cualitativo, ya que su interés se centra en el estudio de los diferentes fenómenos sociales, en visualizar al otro, en interpretar su propio mundo, como afirman Denzin y Lincoln (2005:3), citados por Rodríguez, y Valldeoriola (SF), "una actividad donde el mundo se hace visible... prácticas transformadoras de la cosmovisión, que implican una aproximación interpretativa y naturalista del mundo.

Y toma como punto de partida la Investigación Acción Participante ya que como afirma Fals Borda y Anisur (1991:154), es un proceso de intervención social que propone el análisis de una realidad como forma de conocimiento, además sensibiliza y motiva, al individuo para que transforme, a través de sus propias acciones el entorno inmediato.

Tomando como base la anterior afirmación, se pueden determinar las diferentes acciones que se realizan en el proyecto establecidas en cuatro etapas que se describen a continuación:

I Etapa: La profesora encargada realiza visitas a diferentes centros educativos y hogares de ancianos con el objetivo de conversar en el director o directora, en estas visitas se les informa acerca de los objetivos del proyecto y se les ofrece la posibilidad de que jóvenes universitarios den asistencia académica a estudiantes que requieran apoyo educativo, en el caso de instituciones educativas y adultos mayores en los hogares de ancianos. 
II Etapa: Cada vez que un grupo de estudiantes nuevos ingresen al Trabajo Comunal Universitario (TCU), se realiza con ellos una reunión con la finalidad de darles a conocer los objetivos, metodología y alcances del proyecto. En esta reunión se establecerán grupos de trabajo y se les da a conocer posibles centros educativos a considerar para desarrollar el proyecto. En esta segunda etapa también se les ofrece a los estudiantes universitarios matriculados en el Trabajo Comunal Universitario (TCU) un curso de inducción cuya duración es de 30 horas, esta inducción estará distribuida en cinco semanas aproximadamente y será programada con la persona coordinadora del proyecto y los estudiantes avanzados. En este, se dan a conocer aspectos importantes sobre la diversidad y la educación inclusiva, además les proporcionar información básica sobre la Normativa Legal que determina la Educación Inclusiva y los diferentes trastornos y situaciones que inciden en el aprendizaje de una persona, esto propiciará un trabajo más efectivo de los estudiantes universitarios en los centros educativos.

III Etapa: Los estudiantes se incorporan a los respectivos centros educativos e inician su trabajo de acompañamiento con aquellos estudiantes, que a criterio de los docentes, requieren de apoyos para lograr un aprendizaje exitoso; de la misma forma inician su labor con personas adultas mayores que se encuentran institucionalizados en hogares de ancianos. Uno de los requisitos es que los jóvenes universitarios se presenten en la institución en la cual desarrollan su labor al menos seis horas a la semana.

Una actividad que resulta muy provechosa es la programación, en los centros educativos, de reuniones con padres y madres de familia con el propósito de darles a conocer el proyecto, pedirles su aprobación y colaboración e indicarles los lineamientos que se seguirán. En esta reunión se les solicita que firmen un documento de consentimiento, donde ellos aceptan el trabajo de apoyo para sus hijos

De acuerdo con los grupos interdisciplinarios conformados por los estudiantes universitarios de diferentes carreras, se organizan actividades lúdico-recreativas (caminatas, convivencias, juegos al aire libre, ejercicios, juegos de mesa), no solo con los estudiantes que se les brinda apoyo en el área educativa, sino con toda la población escolar, esto con el objetivo de fomentar el respeto, el trabajo cooperativo, la aceptación del otro y prevenir la exclusión en esta población. En los hogares de ancianos se realizan talleres lúdico recreativos, tipo convivencia, con las personas institucionalizadas y en algunas fechas 
especiales se extienden estas actividades a sus familiares y allegados, lo que resulta sumamente provechoso y muy significativo para toda esta comunidad.

Otra actividad de gran relevancia son los talleres que se programan talleres en las diferentes instituciones con temas relacionados con la diversidad, por ejemplo: El respeto al otro, NO AL BULLING, empatía, resiliencia, etc. O temas que los directores o directoras de los centros educativos soliciten, siempre relacionados con inclusión.

IV Etapa: Se realiza la evaluación de las actividades ejecutadas por medio de reuniones con los estudiantes matriculados en el Trabajo Comunal Universitario (TCU) quienes darán a conocer su punto de vista y harán recomendaciones para realimentar el proyecto. También se evalúa el proyecto solicitando la opinión a los usuarios del proyecto: directores, docentes, padres de familia, estudiantes con alguna deficiencia. Al concluir cada periodo lectivo se elabora un informe en el que se valoran los logros, limitaciones y se enriquece el proyecto tomando en cuenta esta evaluación.

\section{Resultados}

Gracias a la metodología IAP utilizada en el desarrollo del proyecto de TCU “Atención a la diversidad", se ha logrado construir un clima de confianza entre los estudiantes universitarios, quienes comprometidos con su labor se han dedicado a construir espacios de inclusión para niños, niñas, jóvenes y adultos mayores vulnerables a la exclusión, partiendo de las necesidades de estas poblaciones, sin pretender caer en el asistencialismo o la rehabilitación.

La mayoría de las veces, al iniciar sus labores en el Trabajo Comunal Universitario, los jóvenes estudiantes se percibían un tanto inseguros, debido a la poca experiencia y a diferentes prejuicios, sin embargo al compartir espacios de reflexión con sus compañeros y coordinadora del proyecto, adquieren mayor confianza y seguridad, lo que les permitió desarrollar las diferentes actividades (acompañamiento en el aprendizaje, atención individual, talleres, juegos al aire libre, actividades socioculturas y otras), de una manera espontánea y provechosa, no solo para ellos, sino para los beneficiarios. Como lo mencionan claramente Stainback y Stainback y Jackson (1999:23): "la inclusión como práctica construye una auténtica comunidad de tal manera que todos se sienten ligados, aceptados, 
apoyados y en las que cada uno apoyando a otro y fortaleciéndose en el otro logre satisfacer sus propias necesidades educativas".

Al concluir sus 300 horas de trabajo comunal, los jóvenes matriculados en el Trabajo Comunal Universitario (TCU), deben preparar un informe en el que dan a conocer todas las labores realizadas y principalmente los logros y limitaciones que encontraron en el desarrollo de su proyecto. A manera de ejemplo se detallan algunas de las opiniones que los estudiantes han manifestado a la hora de concluir su trabajo y que permiten de una u otra manera comprender que realmente la labor que se ejecuta en este proyecto no solo ha impactado a las poblaciones vulnerabilizadas con las que se trabaja, sino muy especialmente a los jóvenes universitarios quienes ejecutan el proyecto:

P: "Para mí el desarrollo del trabajo comunal universitario significó un espacio de reflexión que me permitió no solo realizar un trabajo que me dejó muchas gratificaciones, sino fue un proceso de aprendizaje paralelo, porque aunque no contaba con la formación específica en educación, logre dar apoyo a dos estudiantes y la relación con ellos fue muy provechosa, esto permite que germine una semilla de la no discriminación y la apertura a distintas formas de enseñanza en cualquier área”

A: A nivel personal el TCU me permitió desarrollar mi creatividad y la iniciativa para desarrollar diferentes temáticas, que aunque son parte de mi formación profesional, no había tenido la oportunidad de poner en práctica, además el compartir con compañeros de otras carreras como educación me generó un nuevo aprendizaje, especialmente en la aplicación de nuevas estrategias para promover el aprendizaje. También generó en mí una gran sensibilización, un compromiso ético y político como futura profesional con los grupos vulnerabilizados, los cuales muchas veces son excluidos y discriminados, es necesario defender los derechos de estas personas y contribuir con la erradicación de estereotipos y prejuicios".

K: En cuanto a mi persona, muy agradecido con Dios por brindarme una oportunidad de crecimiento personal como educador al trabajar directamente con lo que compete al tema de discapacidad, lo que en mi carrera no se abarca a través de un curso. Además, el haberme permitido aprender de cada uno de los procesos llevados a cabo me confrontó con una pincelada de mi futura profesión y el reto que tenemos ante la educación inclusiva. 
JD: Como estudiante universitario, debo admitir que la labor del TCU se convierte en un complemento importante al contenido teórico y práctico de nuestras carreras universitarias. El haber trabajado con grupos tan diversos, en distintas comunidades del país, me permitió conocer una realidad de la cual no estamos enterados. Pude conocer historias y puntos de vista de los cuales aprendí, que me permiten también adquirir mayor sensibilidad antes problemáticas sociales y situaciones a las cuales tal vez no somos afectados directos. Sumado a esto, conocí técnicas de abordaje a grupos y contenidos, que considero me podrían ser útiles en el desempeño de mi profesión.

C: El desarrollar el trabajo comunal "Atención a la Diversidad" al principio me sentí un poco confundida pero al compartir con los niños y jóvenes con necesidades educativas me hizo "perder el miedo y deshacerme de mitos y prejuicios que había construido a través de mi vida, me permitió enfrentarme a ellos y deshacerme de ellos, ahora son una persona diferente, me di cuenta que son seres humanos como nosotros y creo que haciendo el TCU, aprendí lo que no aprendí con todos los cursos universitarios juntos".

SH: Trabajar en las distintas actividades del TCU me hizo comprender que todos los seres humanos somos valiosos, y muchas veces las personas con trastornos de aprendizaje son subestimadas y poco valoradas, esto me hizo meditar como futura docente y como persona, todos tenemos derecho a una educación de calidad y a que se nos respete y se nos valore, realmente la discapacidad está en nosotros que no podemos ver a todas las personas como seres humanos en igualdad de condiciones".

Expresiones como las anteriores dejan entrever que el cambio que se generó en los estudiantes universitarios al desarrollar diferentes actividades inclusivas con estudiantes con discapacidad y con adultos mayores les permitió entender al otro, hacerlo visible, como afirma Gentili (2001).

"La escuela democrática debe contribuir a volver visible lo que la mirada normalizadora oculta. Debe ayudar a interrogar, cuestionar, comprender los factores que han contribuido a la barbarie que supone negar los más elementales derechos humanos y sociales a las grandes mayorías, para ello es necesario aproximarse a ese Otro, no imaginarlo, es reconocerlo". 
Retomando la cita anterior, la Universidad por su carácter humanista debe proveer estos espacios de reflexión, procurar que el universitario construya habilidades que le permitan como futuro profesional incorporarse asertivamente en una sociedad diversa.

Asimismo, al utilizarse la IAP en el desarrollo del proyecto, y al ser esta una metodología que promueve la dialogicidad, las relaciones armoniosas y la confianza de cada uno de los participantes en el proceso, también se ha tomado en cuenta la opinión de los beneficiarios, en este caso directores, docentes, estudiantes y adultos mayores quienes han interactuado con los jóvenes universitarios para conocer de qué manera ha coadyuvado el proyecto en la mejora de su labor o en el avance de los niños, niñas y jóvenes apoyados y han expresado sentirse muy satisfechos. Los docentes que han tenido la experiencia de contar con el apoyo de un estudiante de Trabajo Comunal Universitario (TCU) para un niño o niña de su grupo han manifestado sentirse muy complacidos, consideran que el aporte que hacen los estudiantes universitarios en las aulas de primaria y preescolar es invaluable. Algunos han expresado:

"Mándeme todos los estudiantes que tenga, ojalá pudiera tener por lo menos dos por grupo dando apoyo a los chiquitos". "El trabajo que hacen estos muchachos con los niños es increíble, se perciben los avances académicos y sobre todo el aumento en la confianza". “Ojalá pudiera tener un muchacho o muchacha ayudándole a todos los chiquitos, es increíble cómo avanzan". (Docentes)

Al respecto es importante destacar lo que menciona Freire (1973:32), "la educación es praxis, reflexión y acción, ingredientes necesarios para transformar al mundo" y definitivamente el trabajo realizado por los jóvenes universitarios ha permitido el cuestionamiento de ciertas prácticas pedagógicas por parte de los docentes y la transformación de ellos mismos.

\section{Conclusiones}

Educar en la diversidad significa ejercer los principios de igualdad y equidad a los que todo ser humano tiene derecho, lo que conlleva a desarrollar unas estrategias de 
enseñanza-aprendizaje que personalicen la enseñanza en un marco y dinámica de trabajo para todos.

Es necesario que los jóvenes universitarios, futura fuerza laboral de un país, visualicen la diversidad como un valor, donde cada individuo, sin importar su condición pueda ejercer los principios de igualdad y equidad.

Todo ser humano tiene derecho a recibir una educación de calidad que se ajuste a sus diferencias, de ahí la importancia de poner en práctica estrategias de enseñanza y aprendizaje acordes a la individualidad de cada persona.

Se considera de suma relevancia que los estudiantes universitarios, independientemente de la carrera que cursen, tengan la oportunidad de tomar conciencia y sensibilizarse ante la diversidad, el proyecto ha propiciado experiencias acordes a esta necesidad.

El acompañamiento que los jóvenes universitarios dan a los niños y niñas con alguna deficiencia genera en estos últimos confianza, seguridad en sí mismo y sobre todo un sentimiento de valía que redunda en un mejoramiento en su rendimiento académico y en su socialización.

Los centros educativos, especialmente la Educación Superior deben procurar un plan de estudios que permita a sus estudiantes, como futuros profesionales, no solo concebir la diversidad como un valor, sino que puedan construir estrategias coherentes con este postulado y tener una cosmovisión coherente con la alteridad, donde el otro se hace visible y enriquece la forma en que se percibe el mundo

Utilizar la Investigación Acción Participante en el desarrollo de este proyecto de Trabajo Comunal Universitario ha constituido un pilar fundamental, ya que no solo se realiza un trabajo para proyectarse a la comunidad, sino que se han generado cambios importantísimos en los jóvenes universitarios y en el contexto en el que se involucran, ya sean centros educativos de preescolar, primaria, secundaria u Hogar de Ancianos. 


\section{Referencias Bibliográficas}

Ainscow, Mel y César, Margarida. (2006). Inclusive education ten years after Salamanca: Setting the agenda. European Journal of Psychology of Education, vol. xxi (3), 231-238.

Arnaiz, Pilar. (2003) Educación Inclusiva. Una escuela para todos. Málaga, España. Ediciones Aljibe, SL.

Gentilli, Pablo (2001). Un zapato perdido. Revista Dialnet No. 302

Gobierno de Costa Rica (1998). Código de la niñez y la adolescencia. San José, Costa Rica, la Gaceta No. 26 (6 de febrero, 1998).

Costa Rica. Leyes, decretos (2004). Ley 7600 sobre igualdad de oportunidades para las personas con discapacidad. San José, Costa Rica. Editorama.

Fals Borda, 0. y Anisur, M. Rahman. (1991). Acción y Conocimiento. Como romper el Monopolio con Investigación Acción Participativa. Bogotá, Colombia. Cinep.

Freire, Paulo. (1973). Pedagogía del oprimido. México. Siglo Veintiuno Editores.

Rodríguez, David y Valldeoriola, Jordi (SF). Metodología de la Investigación. Universidad Oberta de Catalunya. Recuperado (3.08.2017) http://zanadoria.com/syllabi/m1019/mat_cast-nodef/PID_00148556-1.pdf

Stainback, Susan., Stainback, William y Jackson, James (1999). Hacia las aulas inclusivas. Madrid, España. Narcea.

UNESCO (2009). Directrices sobre políticas de inclusión en la educación. Paris. Organización de las naciones Unidas para la Educación, la Ciencia y la Cultura.

Vicerrectoría de Acción Social, Universidad de Costa Rica (2017). Portal de Acción Social. Recuperado (26.05.2017) de http://accionsocial.ucr.ac.cr/trabajo-comunal

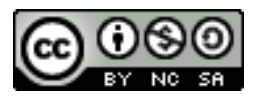

